\title{
EVALUATION AND CORRECTION OF SYSTEMATIC EFFECTS IN A SIMULTANEOUS 3-AXIS VIBRATION CALIBRATION SYSTEM
}

\author{
A. Prato $^{1}$, F. Mazzoleni ${ }^{1}$, A. Schiavi ${ }^{1}$ \\ ${ }^{1}$ INRiM - National Institute of Metrological Research, Torino, Italy, a.schiavi@inrim.it
}

\begin{abstract}
:
This paper presents a calibration method, recently realized at INRIM, suitable for the calibration of 3axis accelerometers in frequency domain. The procedure, allows to simultaneously evaluate the main and transverse sensitivities on three axes by means of a single-axis vibration excitation of inclined planes. Nevertheless, the excitation system is subjected to spurious motions mainly due to the vibrational modes of the inclined planes and to the horizontal motions of the shaker. In order to provide the proper sensitivities to the 3 -axis sensors, the evaluation of systematic effects is experimentally carried out and the related correction is proposed.
\end{abstract}

Keywords: calibration, 3-axis accelerometer, systematic effects

\section{INTRODUCTION}

The 3-axis accelerometers, especially low-cost unconventional-shaped transducers, such as MEMS sensors, are largely used in a wide range of advanced industrial, environmental, energy and medical applications, and in particular within extensive sensor and multi-sensor networks [e.g., $1-10]$. For example, in the context of Industry 4.0, a huge number of sensors is needed for an effective implementation of smart factories, learning machine and intelligent manufacturing systems, as well as for traditional application such as early failure detection and predictive maintenance; low-power devices and battery-operated systems are practical and useful in IoT applications, such as for smart cities, for accurate navigation/positioning systems and in environmental monitoring and survey; moreover, accurate measurements are of paramount importance in medical applications, in remote surgery and remote diagnoses. The possibility to have many accurate, low-power consuming and low-cost sensors present undoubted advantages, in terms of costs reduction and energy saving, in the control processes, monitoring or measurements and being flexible in providing enhanced data collection, automation and operation. By way of example, the calibration of digital MEMS sensors, with the associated uncertainty budget, allows to ensure traceability and measurement accuracy of nodes in sensor networks, as well as in other innovative implementations. Moreover, the evolving improvement of the technical performance and the reliability of MEMS sensors are emerging quality attributes of interest for manufacturers, costumers and end-users.

However, in the particular case of digital MEMS accelerometers, the sensitivity is generally provided by manufacturer without traceable calibration methods and is obtained in static conditions, whereas dynamic response, as a function of frequency, is often barely known or completely disregarded. Given this condition, a simultaneous 3 -axis vibration calibration system with a single axis excitation to be exploitable by manufacturers is proposed. This paper deals with the evaluation of systematic effects of this system.

\section{DESCRIPTION OF THE WORK}

Traceable calibration methods for digital sensors, and smart sensors, in metrological terms [11 - 13], including sensitivity parameter and an appropriate uncertainty evaluation, are necessary in order to consider low-cost and low-power consuming accelerometers as actual measurement devices in frequency domain [14].

The calibration system, developed at INRiM, allows to simultaneous evaluate the main and the transverse sensitivity, in frequency domain, of 3-axis accelerometers, by means of a single-axis vibration excitation, by using inclined planes rigidly fixed to the vibrating table $[14,15]$. The calibration procedure is based on the comparison to a reference transducer (in analogy to ISO 16063-21[16]). A preliminary version of the system was previously investigated for the characterization of analog MEMS accelerometers performance in operative conditions [17 - 23].

Measurements are performed at a nearly constant amplitude of $10 \mathrm{~m} \cdot \mathrm{s}^{-2}$, from $5 \mathrm{~Hz}$ up to $3 \mathrm{kHz}$. The mechanical calibration system, composed of the shaker and the inclined planes (with tilt angles of $15^{\circ}$, $35^{\circ}, 55^{\circ}$ and $75^{\circ}$ ), is characterized in order to take into account systematic effects. Similar procedure is also applied to the shaker at $0^{\circ}$ and $90^{\circ}$. 
Measurements of systematic effects is carried out by means of a Laser-Doppler vibrometer. The detailed uncertainty budged is evaluated according to GUM [24].

\section{CALIBRATION SET-UP}

The calibration set-up here proposed, consisting of a single-axis vibrating table on which aluminum inclined planes are screwed, allows to generate a projection of the reference acceleration along three axes simultaneously. A single vertical sinusoidal acceleration at nearly-constant amplitude acts as reference acceleration $a_{r e f}$ along the vertical $z$ '-axis of the system. In this way, accelerations of proportional amplitudes, are simultaneously generated on the inclined surface plane, along the three axes.

In figure 1, the geometrical principle of the proposed method on which the 3-axis accelerometer is fixed during calibration, is schematically depicted.

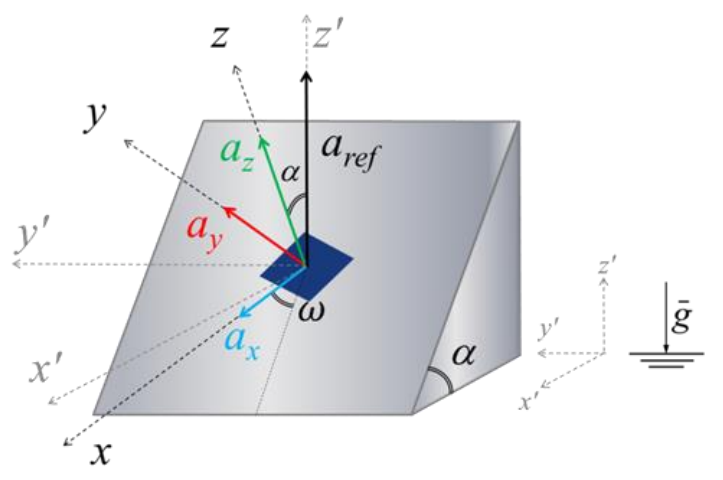

Figure 1: Inclined plane - scheme

From simple trigonometrical laws, the reference accelerations detected by the sensor in calibration, along its three sensitive axes, are expected to be:

$$
\begin{aligned}
& a_{x, \text { theor }}=\left|a_{\text {ref }} \sin (\alpha) \cos (\omega)\right| \\
& a_{y, \text { theor }}=\left|a_{\text {ref }} \sin (\alpha) \sin (\omega)\right| \\
& a_{z, \text { theor }}=\left|a_{\text {ref }} \cos (\alpha)\right|
\end{aligned}
$$

where, $\alpha$ is the tilt angle, $\omega$ is the angle of rotation, $a_{r e f}$ is the Root Mean Square (RMS) reference acceleration along the vertical $z$ '-axis of the system, and $a_{x, \text { theor }}, a_{y, \text { theor }}, a_{z, \text { theor }}$ are the RMS reference accelerations spread along $x$-, $y$ - and $z$-axis of the MEMS accelerometer in calibration.

In the experimental set-up, the inclined plane is screwed on the vertical vibrating table $(P C B$ Precision Air Bearing Calibration Shaker), and the 3axis accelerometer is fixed to the inclined plane and located along the vertical axis of excitation. The experimental configuration is shown in figure 2 .

The acceleration along vertical $z^{\prime}$-axis $a_{r e f}$ is measured by a single axis reference transducer $(P C B$ model 080A199/482A23), calibrated according to
ISO 16063-11:1999 [25], against INRiM primary standard, located within the stroke of the shaker and is acquired by an acquisition board NI 4431 (sampling rate of $50 \mathrm{kHz}$ ) integrated in the PC and processed through LabVIEW $^{\circledR}$ software to provide the RMS reference value in $\mathrm{m} \mathrm{s}^{-2}$.

The digital MEMS output is acquired by an external microcontroller at a maximum sampling rate of $6.660 \mathrm{kHz}$ and saved as binary files.

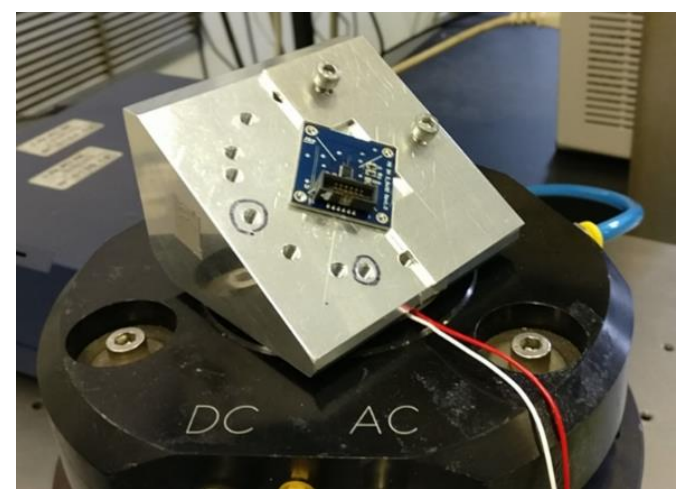

Figure 2: The calibration set-up: the MEMS fixed to the inclined plane on the vibrating table.

\section{EVALUATION OF SYSTEMATIC EFFECTS AND CORRECTION}

As described in the previous Section, the reference accelerations along MEMS accelerometer sensitivities axes are given by equations (1)-(3), by using trigonometrical laws. However, in dynamic conditions, systematic effects caused by spurious oscillating components along the three axes of the reference system ( $x^{\prime}-, y^{\prime}-$ and $\left.z^{\prime}\right)$ at MEMS position need to be taken into account. These spurious components affect the actual reference accelerations $a_{\text {ref }}$ splitted on the three sensitivity axes of the MEMS. In Figure 3, a schematic representation of the occurring phenomenon is shown.

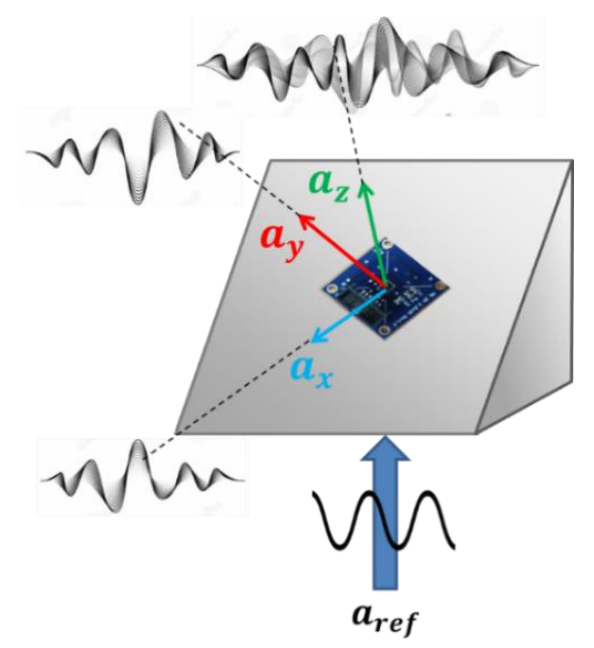

Figure 3: Representation of the spurious oscillating components combination along the three axes of the MEMS during the calibration. 
Such components are mainly due to the vibrational modes of the inclined aluminum planes and due to small but not negligible horizontal motions of the shaker. Each spuroius component along reference system $x^{\prime}-, y^{\prime}-$ and $z^{\prime}$-axis, has to be decomposed along the 3-axis accelerometer $x$-, $y$ - and $z$-axis and summed to the reference accelerations $a_{x, \text { theor }}, a_{y, \text { theor }}$, $a_{z, \text { theor }}$ according to wave interference laws. The actual decomposition of the spurious components, acting along the three axes of the reference system, is schematically depicted in Figure 4.

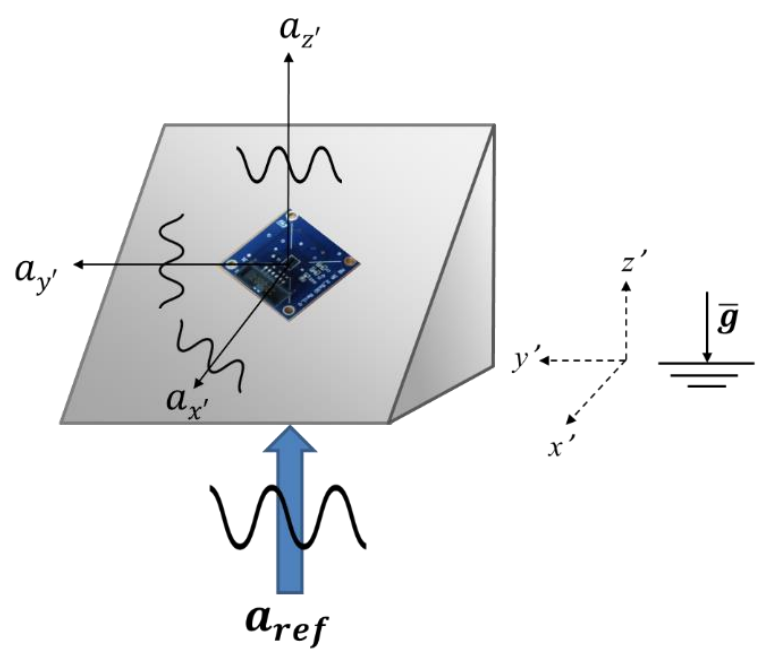

Figure 4: Representation of the spurious oscillating component decomposition, along the three axis of the reference system, at a given frequency.

By way of example, considering the general case of four overlapping waves, $E_{1}=E_{1,0} e^{i(2 \pi f t)}, E_{2}=$ $E_{2,0} e^{i\left(2 \pi f t+\varphi_{2}\right)} \quad, \quad E_{3}=E_{3,0} e^{i\left(2 \pi f t+\varphi_{3}\right)} \quad, \quad E_{4}=$ $E_{4,0} e^{i\left(2 \pi f t+\varphi_{4}\right)}$, oscillating at a same frequency $f$, with different amplitudes and phase differences with respect to the reference signal $E_{l}$, along a particular direction, their interference can be opportunely defined according to equation (4), where $E_{2}, E_{3}$ and $E_{4}$ are the amplitude- and phase-dependant spurious components along $x-, y$ - and $z$-axis of the MEMS.

$$
E_{\text {tot }}=\left|E_{1,0}+E_{2,0} e^{i \varphi_{2}}+E_{3,0} e^{i \varphi_{3}}+E_{4,0} e^{i \varphi_{4}}\right|
$$

In this way, it is possible to correct reference theoretical accelerations along MEMS axes in equations (1)-(3), into $a_{x}, a_{y}, a_{z}$ of equations (8)-(10), where $a_{x}{ }^{\prime}$, syst $, a_{y^{\prime}, \text { syst }}, a_{z}$, ,syst and $\varphi_{x^{\prime}, \text { syst }}, \varphi_{y^{\prime}, \text { syst }}, \varphi_{z^{\prime}, \text { syst }}$ are, respectively, the amplitudes and the phase differences as shown in equations (5)-(7), with respect to the reference signal $a_{r e f}$, of the spurious components along $x^{\prime}-, y^{\prime}$ - and $z^{\prime}$-axis. As it will be shown in Section 5, the amplitude of spurious components vary as a function of frequency between $0.1 \%$ and $10 \%$ of the reference acceleration $a_{r e f}$. Experimental evaluation of systematic effects due to spurious components is carried out by means of a laser-Doppler velocimeter (Polytec OFV 505).

Amplitude and phase measurements along the $x$ '-, $y^{\prime}$ - and $z^{\prime}$-axis of the reference system are evaluated for each inclined plane and for all frequencies, at reference vertical amplitude of $10 \mathrm{~m} \mathrm{~s}^{-2}$. Laser signal, during measurements of spurious components amplitude, is acquired by a NI 4431 board (sampling rate of $50 \mathrm{kHz}$ ) integrated into the $\mathrm{PC}$, while measurement of phase differences between reference acceleration and spurious components are measured by means of a dynamic signal analyzer (KEYSIGHT 35670A).

Since the digital MEMS accelerometer is too small to be used as a reflective surface, the beam spot of the laser directly hits a small aluminum triangular-based parallelepiped located at MEMS position and fixed to the different inclined planes, as shown in figure 5 . The volume of the triangular-based parallelepiped is around $0.5 \mathrm{~cm}^{3}$, which is $0.6 \%$ of the total volume of the inclined plane, i.e., negligible with respect to the total mass.

The values of the measured acceleration amplitudes $a_{x}, a_{y^{\prime}}$ and $a_{z}$ ' along $x^{\prime}-, y^{\prime}$ - and $z^{\prime}$-axis are related to the actual systematic effects acting on the axes of the reference system, and are expressed, as a function of frequency and experimental phase-shift, by the following equations:

$$
\begin{aligned}
& a_{x^{\prime}}=\left|a_{x^{\prime}, \text { theor }}+a_{x^{\prime}, \text { syst }} \cdot e^{i\left(2 \pi f t+\varphi_{x^{\prime}, \text { syst }}\right)}\right| \\
& a_{y^{\prime}}=\left|a_{y^{\prime}, \text { theor }}+a_{y^{\prime}, \text { syst }} \cdot e^{i\left(2 \pi f t+\varphi_{y^{\prime}, \text { syst }}\right)}\right| \\
& a_{z^{\prime}}=\left|a_{z^{\prime}, \text { theor }}+a_{z^{\prime}, \text { syst }} \cdot e^{i\left(2 \pi f t+\varphi_{z^{\prime}, \text { syst }}\right)}\right|
\end{aligned}
$$

where $a_{x}$,theor and $a_{y}$, theor are 0 (i.e., no acceleration is generated along the horizontal system plane $x^{\prime}-y^{\prime}$ ) and the vertical component $a_{z^{\prime}, t h e o r} \equiv a_{\text {ref }}$.

In Figure 5 the experimental method used to quantify the spurious components from accelerations $a_{x^{\prime}}, a_{y^{\prime}}$ and $a_{z^{\prime}}$, and the related phase-shift $\varphi_{x^{\prime}}, \varphi_{y^{\prime}}$ and $\varphi_{z}$, with respect to the reference acceleration $a_{r e f}$, acting along the vertical axis $z$, is shown.

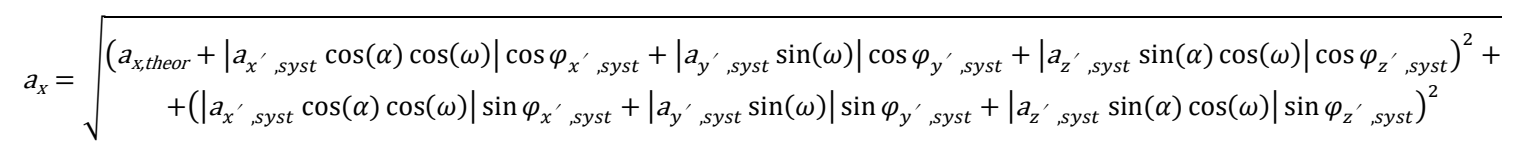




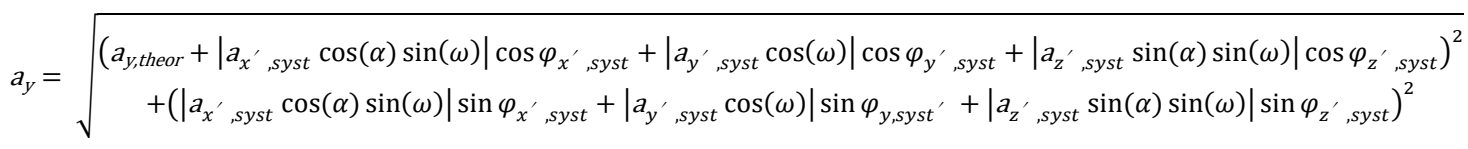

$$
\begin{aligned}
& a_{z}=\sqrt{\begin{array}{c}
\left(a_{z, \text { theor }}+\left|a_{x^{\prime}, \text { syst }} \sin (\alpha)\right| \cos \varphi_{x^{\prime}, \text { syst }}+\left|a_{z^{\prime}, \text { syst }} \cos (\alpha)\right| \cos \varphi_{z^{\prime}, \text { syst }}\right)^{2}+ \\
+\left(\left|a_{x^{\prime}, \text { syst }} \sin (\alpha)\right| \sin \varphi_{x^{\prime}, \text { syst }}+\left|a_{z^{\prime}, \text { syst }} \cos (\alpha)\right| \sin \varphi_{z^{\prime}, \text { syst }}\right)^{2}
\end{array}}
\end{aligned}
$$
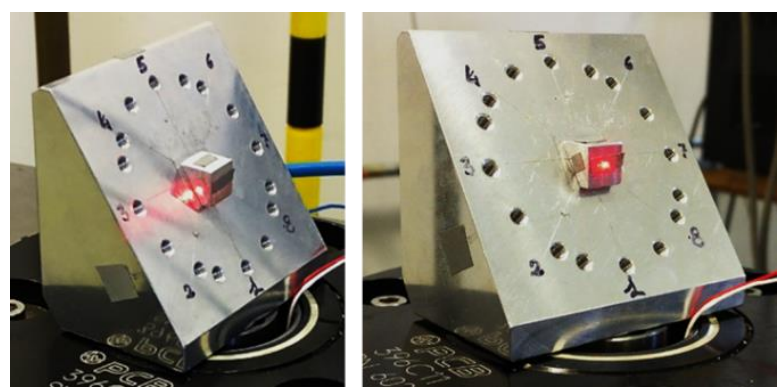

Figure 5: The laser beam hitting the aluminum triangularbased parallelepiped located at the MEMS position.

\section{EXPERIMENTAL RESULTS}

In the graphs of Figures $6-9$, the values of the amplitudes of $a_{x}, a_{y}$, and $a_{z}$, are normalized with respect to the reference acceleration $a_{r e f}$ amplitude. Mesurement are performed from $5 \mathrm{~Hz}$ and $3 \mathrm{kHz}$.

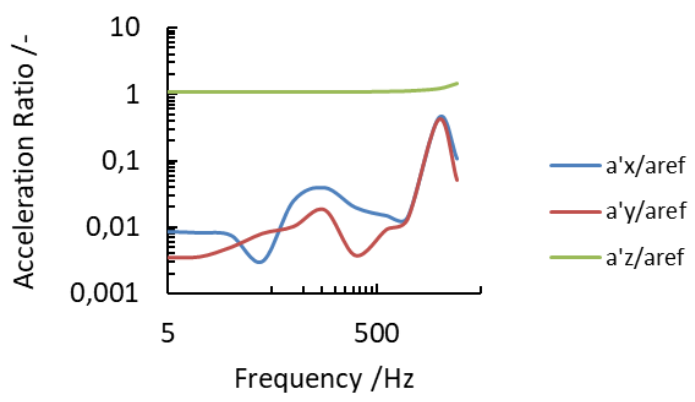

Figure 6: Normalized accelerations along $x^{\prime}-, y^{\prime}-$ and $z^{\prime}-$ axis at $15^{\circ}$ of tilt angle.

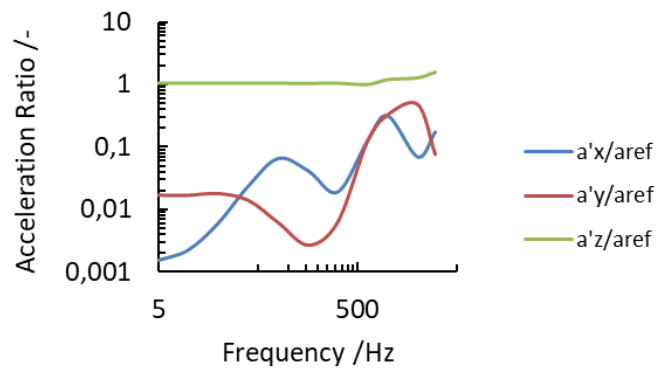

Figure 7: Normalized accelerations along $x^{\prime}-, y^{\prime}-$ and $z^{\prime}-$ axis at $35^{\circ}$ of tilt angle.

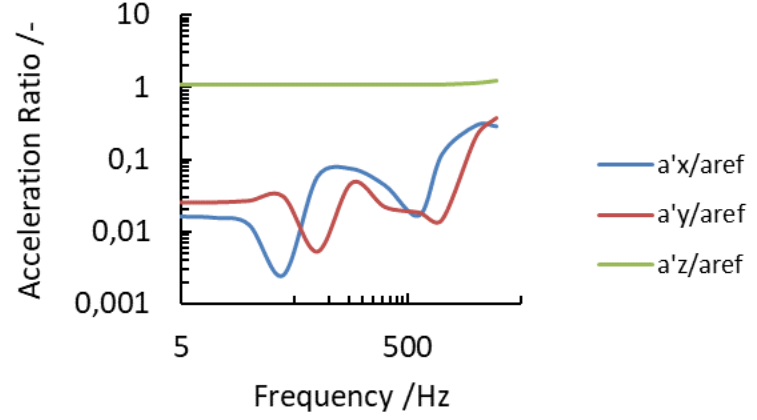

Figure 8: Normalized accelerations along $x^{\prime}-, y^{\prime}-$ and $z^{\prime}-$ axis at $55^{\circ}$ of tilt angle.

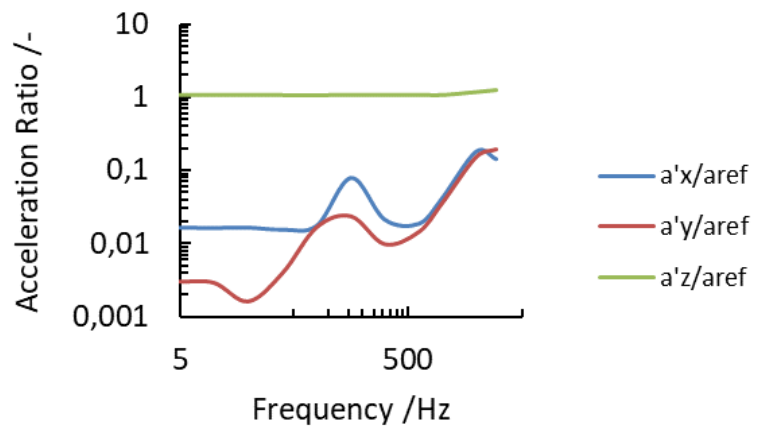

Figure 9: Normalized accelerations along $x^{\prime}-, y^{\prime}-$ and $z^{\prime}-$ axis at $75^{\circ}$ of tilt angle.

The experimental values of the spurious components amplitude of accelerations $a_{x}, a_{y^{\prime}}$ and $a_{z}$, along the axes of the system, are combined in order to calculate the values of systematic effects due to the acceleration amplitudes $a_{x, \text {,syst }}, a_{y^{\prime}, \text { syst }}$ and $a_{z^{\prime}, \text { syst }}$, along the MEMS sensitivity axes.

In the graphs of the Figures $10-13$, the experimental values of phase-shift $\varphi_{x}, \varphi_{y}$, and $\varphi_{z}$, with respect to the reference acceleration $a_{r e f}$, acting along the vertical axis $z$, are shown. In this case the values of phase-shift allow to evaluate the phase differences, with respect to the reference signal $a_{\text {ref }}$, in terms of $\varphi_{x, \text { syst }}, \varphi_{y^{\prime}, \text { syst }}$ and $\varphi_{z^{\prime}, \text { syst. }}$. Since $\varphi_{z^{\prime}, \text { syst }}$ is close to $0^{\circ}$ in every configuration, it is not shown in the graphs. 


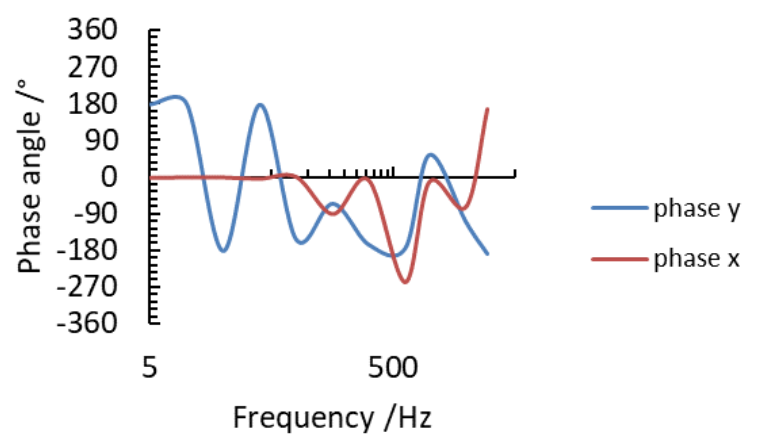

Figure 10: Phase-shifts on the $x^{\prime}-y^{\prime}$ horizontal plane, with respect to the vertical axis $z^{\prime}$, at $15^{\circ}$ of tilt angle.

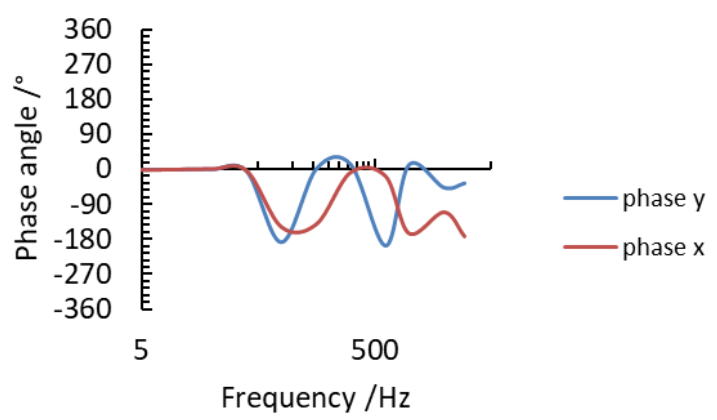

Figure 11: Phase-shifts on the $x^{\prime}-y^{\prime}$ horizontal plane, with respect to the vertical axis $z$, at $35^{\circ}$ of tilt angle.

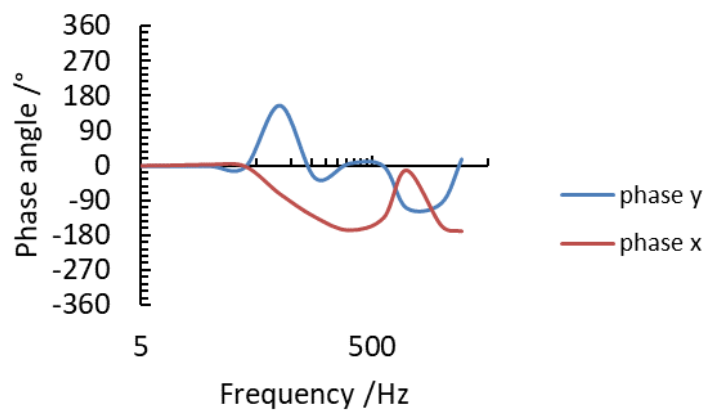

Figure 12: Phase-shifts on the $x^{\prime}-y^{\prime}$ horizontal plane, with respect to the vertical axis $z^{\prime}$, at $55^{\circ}$ of tilt angle.

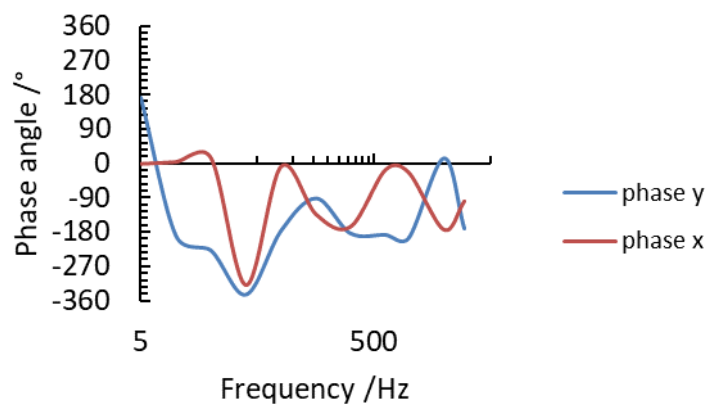

Figure 13: Phase-shifts on the $x^{\prime}-y^{\prime}$ horizontal plane, with respect to the vertical axis $z^{\prime}$, at $75^{\circ}$ of tilt angle.

Once quantified the acceleration amplitudes $a_{x}$, $a_{y}$, and $a_{z}$, and the values of the phase-shift $\varphi_{x}, \varphi_{y}$, and $\varphi_{z}$, the reference theoretical accelerations along MEMS axes in equations (1)-(3), can be opportunely expressed into $a_{x}, a_{y}, a_{z}$ of equations (8)-(10), by taking into account the systematic effects due to the vibrational modes of the inclined planes and to horizontal motions of the shaker. In particular, it can be observed an increasing of acceleration amplitudes, as a function of frequency, along the horizontal axes $x^{\prime}, y^{\prime}$ and also along vertical axis $z^{\prime}$, this last mainly due to resonant modes. Moreover, the lateral motions of the shaker, occurring around $80 \mathrm{~Hz}$, are presumably the cause of the large phase-shifts observed at low frequencies, as depicted in Figure 10 - 13; these motions are independent from the resonant modes of the inclined planes, but they are affecting in any case, the whole behavior of the inclined plane, in terms of amplitude and phase differences. On the other hand, the analysis of the systematic effects, is an aggregation of both shaker spurious motions and resonant modes.

Standard uncertainties associated to the amplitude of the spurious components, $u\left(a_{x^{\prime}, \text { syst }}\right), u\left(a_{y^{\prime}, s y s t}\right)$, $u\left(a_{z, \text { syst }}\right)$, are considered as type $\mathrm{B}$ uncertainty contributions with an average error of $\pm 0.0025 \mathrm{~m} \mathrm{~s}^{-2}$, from three repeated measurements, and a uniform rectangular distribution. Standard uncertainties associated to the phase difference due to the spurious components, $u\left(\varphi_{x^{\prime}, \text { syst }}\right), \quad u\left(\varphi_{y^{\prime}, \text { syst }}\right), \quad u\left(\varphi_{z^{\prime}, \text { syst }}\right), \quad$ are considered as type A uncertainty contributions with a maximum standard deviation of $2^{\circ}$ from five repeated measurements, as shown in [14].

This correction allows to univocally define the actual projection of the reference acceleration $a_{\text {ref }}$ on the three axes, thus the "standard" calibration can be achieved, by comparison to the reference transducer within the stroke of the shaker and it can be finally related to the primary Standard, as declared.

\section{SUMMARY}

In this paper is presented a technical insight on the calibration system, recently realized at INRIM, suitable for the calibration of 3-axis accelerometers in frequency domain. The procedure, allows to simultaneously evaluate the main and transverse sensitivities on three axes by means of a single-axis vibration excitation of inclined planes. The mechanical calibration system, composed of the shaker and the inclined planes, is characterized in order to take into account systematic effects occuring during dynamical excitation. The evaluation of systematic effects, due to the vibrational modes of the inclined aluminum planes and due to small but not negligible horizontal motions of the shaker, are carried out from $5 \mathrm{~Hz}$ up to $3 \mathrm{kHz}$ at an amplitude of $10 \mathrm{~m} \mathrm{~s}^{-2}$. The amplitudes of the acceleration spurious components $a_{x}, a_{y}$, and $a_{z}$, and the values of the phase-shift $\varphi_{x}, \varphi_{y^{\prime}}$ and $\varphi_{z}$, with respect to the reference acceleration $a_{r e f}$, acting along the vertical axis $z^{\prime}$, are accurately measured by means of a LaserDoppler vibrometer. This correction allows to 
univocally define the actual projection of the reference acceleration $a_{\text {ref }}$ on the three axes, thus the "standard" calibration can be achieved and related to the primary Standard.

\section{NOTE}

This work has to be considered as an addendum to the paper: "Prato, A., Mazzoleni, F., \& Schiavi, A. (2020). Traceability of digital 3-axis MEMS accelerometer: simultaneous determination of main and transverse sensitivities in the frequency domain. Metrologia, 57(3), 035013" [14], which shows the detailed extension of the determination of the systematic effects of the calibration system.

\section{REFERENCES}

[1] Noel, A. B., Abdaoui, A., Elfouly, T., Ahmed, M. H., Badawy, A., \& Shehata, M. S. (2017). Structural health monitoring using wireless sensor networks: A comprehensive survey. IEEE Communications Surveys \& Tutorials, 19(3), 1403-1423.

[2] Dehkordi, S. A., Farajzadeh, K., Rezazadeh, J., Farahbakhsh, R., Sandrasegaran, K., \& Dehkordi, M. A. (2020). A survey on data aggregation techniques in IoT sensor networks. Wireless Networks, 26(2), 1243-1263.

[3] Deng, X., Jiang, Y., Yang, L. T., Lin, M., Yi, L., \& Wang, M. (2019). Data fusion based coverage optimization in heterogeneous sensor networks: A survey. Information Fusion, 52, 90-105.

[4] Adeel, A., Gogate, M., Farooq, S., Ieracitano, C., Dashtipour, K., Larijani, H., \& Hussain, A. (2019). A survey on the role of wireless sensor networks and IoT in disaster management. In Geological Disaster Monitoring Based on Sensor Networks (pp. 57-66). Springer, Singapore.

[5] Ge, X., Han, Q. L., Zhang, X. M., Ding, L., \& Yang, F. (2019). Distributed event-triggered estimation over sensor networks: A survey. IEEE transactions on cybernetics.

[6] Kumar, D. P., Amgoth, T., \& Annavarapu, C. S. R. (2019). Machine learning algorithms for wireless sensor networks: A survey. Information Fusion, 49, 1-25.

[7] Kandris, D., Nakas, C., Vomvas, D., \& Koulouras, G. (2020). Applications of wireless sensor networks: an upto-date survey. Applied System Innovation, 3(1), 14.

[8] Priyadarshi, R., Gupta, B., \& Anurag, A. (2020). Deployment techniques in wireless sensor networks: a survey, classification, challenges, and future research issues. The Journal of Supercomputing, 1-41.

[9] Zareei, M., Vargas-Rosales, C., Anisi, M. H., Musavian, L., Villalpando-Hernandez, R., Goudarzi, S., \& Mohamed, E. M. (2019). Enhancing the Performance of Energy Harvesting Sensor Networks for Environmental Monitoring Applications. Energies, 12(14), 2794.

[10] Carminati, M., Kanoun, O., Ullo, S. L., \& Marcuccio, S. (2019). Prospects of Distributed Wireless Sensor Networks for Urban Environmental Monitoring. IEEE Aerospace and Electronic Systems Magazine, 34(6), 4452.

[11] Bruns, T., \& Eichstädt, S. (2018, August). A smart sensor Concept for traceable dynamic measurements. In Journal of Physics: Conference Series (Vol. 1065, No. 21, p. 212011). IOP Publishing.

[12] Dorst, T., Ludwig, B., Eichstädt, S., Schneider, T., \& Schütze, A. (2019, May). Metrology for the factory of the future: towards a case study in condition monitoring. In 2019 IEEE International Instrumentation and Measurement Technology Conference (I2MTC) (pp. 1-5). IEEE.

[13] Seeger, B., Bruns, T., \& Eichstädt, S. (2019). Methods for dynamic calibration and augmentation of digital acceleration MEMS sensors. In 19th International Congress of Metrology (CIM2019)(p. 22003). EDP Sciences.

[14] Prato, A., Mazzoleni, F., \& Schiavi, A. (2020). Traceability of digital 3-axis MEMS accelerometer: simultaneous determination of main and transverse sensitivities in the frequency domain. Metrologia, 57(3), 035013.

[15] Schiavi A, Mazzoleni F and Germak A 2015 Simultaneous 3-axis MEMS accelerometer primary calibration: description of the test-rig and measurements XXI IMEKO World Congress on Measurement in Research and Industry 30 2161-2164

[16] ISO 16063-21 2003 Methods for the calibration of vibration and shock transducers - Part 21: Vibration calibration by comparison to a reference transducer (Geneva: International Organization for Standardization).

[17] D'Emilia G, Gaspari A, Natale E, Mazzoleni F and Schiavi A 2018 Calibration of tri-axial MEMS accelerometers in the low-frequency range - Part 1: Comparison among methods Journal of Sensors and Sensor Systems 7(1) 245257

[18] D'Emilia G, Gaspari A, Natale E, Mazzoleni F and Schiavi A 2018 Calibration of tri-axial MEMS accelerometers in the low-frequency range - Part 2: Uncertainty assessment Journal of Sensors and Sensor Systems 7(1) 403-410.

[19] G. D'Emilia, A. Gaspari, F. Mazzoleni, E. Natale, A. Prato, A. Schiavi, 2020 Metrological characterization of MEMS accelerometers by a LDV, AIVELA.

[20] A. Prato, F. Mazzoleni and A. Schiavi, "Metrological traceability for digital sensors in smart manufacturing: calibration of MEMS accelerometers and microphones at INRiM," 2019 IEEE International Workshop on Metrology for Industry 4.0 and IoT, 371-375, 2019.

[21] M. Galetto, A. Schiavi, G. Genta, A. Prato and F. Mazzoleni, "Uncertainty evaluation in calibration of lowcost digital MEMS accelerometers for advanced manufacturing applications," CIRP Annals 68, 535-538, 2019.

[22] A. Schiavi, A. Prato, F. Mazzoleni, G. D’Emilia, A. Gaspari, E. Natale, "Calibration of digital 3-axis MEMS accelerometers: A double-blind «multi-bilateral» comparison", 2020 IEEE International Workshop on Metrology for Industry 4.0 and IoT.

[23] A. Prato, A. Schiavi, F. Mazzoleni, A. Touré, G. Genta, M. Galetto, "A reliable sampling method to reduce large set of measurements: a case study on calibration of digital 3-axis MEMS accelerometers" 2020 IEEE International Workshop on Metrology for Industry 4.0 and IoT.

[24] JCGM 1002008 Evaluation of Measurement Data Guide to the Expression of Uncertainty in Measurement (GUM), Joint Committee for Guides in Metrology, Sèvres, France.

[25] ISO 16063-11 1999 Methods for the calibration of vibration and shock transducers - Part 11: Primary vibration calibration by laser interferometry (Geneva: International Organization for Standardization. 